analogous fashion but lead to very messy formulae, which furthermore give no additional stable information.

4. Finally a word concerning the proof of Theorem I. It is a known result that when $X=$ point, then $K O\{S(E)\}=K O\left(S^{8 n}\right)$ is generated by 1 and $y$. (See $[2]$ ). Hence (2.1) proves the first statement of Theorem I whenever $E$ is trivial. Now an inductive Meyer-Vietoris argument yields the general case.

\title{
BIBLIOGRAPHY
}

1. F. Adams, Vector-fields on spheres, Bull. Amer. Math. Soc. 68 (1962), 39-41.

2. M. Atiyah and F. Hirzebruch, Vector-bundles and homogeneous spaces, Proc. Sympos. Pure Math. Vol. 3, pp. 7-38, Amer. Math. Soc., Providence, R. I., 1961.

3. A. Borel and J. P. Serre, Le théorème de Riemann Roch (d'apres Grothendieck), Bull. Soc. Math. France 86 (1958), 97-136.

4. R. Bott, Some remarks on the periodicity theorems, Colloque de Topologie, Lille, 1959, Bull. Soc. Math. France.

HARVARD UNIVERSITY

\section{A CONNECTION BETWEEN TAUBERIAN THEOREMS AND NORMAL FUNCTIONS}

BY G. T. CARGO 1

Communicated by J. L. Doob, March 18, 1962

The purpose of this note is to point out that certain Tauberian theorems follow immediately from some recent research of LehtoVirtanen and Bagemihl-Seidel.

Let $D$ denote the open unit disk, let $C$ denote the unit circumference, and let $\rho\left(z_{1}, z_{2}\right)$ denote the non-Euclidean hyperbolic distance between the points $z_{1}$ and $z_{2}$ in $D$.

THeorem. Suppose that $f(z)=\sum a_{n} z^{n}$ and that $n\left|a_{n}\right|$ $\leqq M(n=1,2, \cdots)$ for some constant $M$. Further, suppose that $\left\{z_{n}\right\}$ is a sequence of points in $D$ converging to a point $\zeta$ in $C$ with the property that $\rho\left(z_{n}, z_{n+1}\right) \rightarrow 0$ as $n \rightarrow \infty$. Then, if $f\left(z_{n}\right) \rightarrow c$ as $n \rightarrow \infty$, the series $\sum a_{n} \zeta^{n}$ converges to the sum $c$.

Proof. The hypothesis implies that $\left|f^{\prime}(z)\right| \leqq M /(1-|z|)$. Consequently, $\rho(f(z))|d z| \leqq 2 M d \sigma(z)$ holds for all $z$ in $D$ where $\rho(f(z))$ $=\left|f^{\prime}(z)\right| /\left(1+|f(z)|^{2}\right)$ denotes the spherical derivative of $f$ and $d \sigma(z)$

${ }^{1}$ National Academy of Sciences-National Research Council Postdoctoral Research Associate on leave from Syracuse University. 
$=|d z| /\left(1-|z|^{2}\right)$ denotes the hyperbolic element of length. From this we infer at once (see [2, Theorem 3$]$ ) that $f$ is normal in the sense of Lehto and Virtanen; and, invoking a theorem of Bagemihl and Seidel [1, Theorem 2], we conclude that $f$ has the angular limit $c$ at $\zeta$. The theorem now follows from Littlewood's Tauberian theorem for radial approach.

The theorem contains the Hardy-Littlewood Tauberian theorem for curvilinear approach as a special case.

It is now obvious that one can formulate and prove a number of Tauberian theorems by making use of various known properties of normal functions. Conversely, known Tauberian theorems will sometimes suggest properties of normal functions. For example, the fact that a holomorphic function in $D$ having a finite Dirichlet integral is normal yields at once an extension of a familiar Tauberian theorem.

The author will discuss these matters in more detail elsewhere.

\section{REFERENCES}

1. F. Bagemihl and W. Seidel, Sequential and continuous limits of meromorphic functions, Ann. Acad. Sci. Fenn. Ser. A I 280 (1960), 17 pp.

2. O. Lehto and K. I. Virtanen, Boundary behaviour and normal meromorphic functions, Acta Math. 97 (1957), 47-65.

SyRACUSE UNIVERSITY AND

National Bureau of Standards 